\title{
Performance of a Spread Spectrum Packet Radio Network Link in a Poisson Field of Interferers
}

\author{
Elvino S. Sousa, Member, IEEE
}

\begin{abstract}
Results on the modeling of interference in a radio communication network and performance measures for the link as a function of distance are presented. It is assumed that a transmitter-receiver pair in a radio network is affected by a set of interferers, using the same modulation and power, whose positions are modeled as a Poisson field in the plane. Assuming a $1 / r^{\gamma}$ propagation power loss law, the probability distributions for the noise at the receiver are found to be the stable distributions. Results are given for the probability of symbol error and link capacity as a function of the distance between the transmitter and receiver for direct sequence and frequency hopping spread spectrum schemes. It is found that the frequency hopping schemes are inherently superior and their performance is not dependent on the synchronization of the hopping times for the different users.
\end{abstract}

Index Terms-Spread spectrum, multiple access, frequency hopping, radio networks, stable distributions.

\section{INTRODUCTION}

M ANY PROBLEMS arise in radio network research where the distribution of network self-interference needs to be obtained [1]-[4]. In a typical situation a terminal transmits a packet to a destination at a distance $R$ and we need to evaluate the probability that the transmission is successful. The success of the transmission depends on two factors: first, since there is a limit on the number of signals that a receiver can receive simultaneously, the receiver must have an open channel (or port) that can receive the packet; second, the interference at the receiver must be sufficiently low, in some sense. In order to account for the second factor we must characterize the interference at the receiver. The interference at a location in the network is composed of two components: network self-interference, or interference due to other transmitting terminals; and external interference such as thermal noise and interference from other systems. The interference signals at all points of the network may be collectively modeled as a time varying stochastic field in some region of the plane (for planar networks.) Hence, to

Manuscript received February 1988; revised. This work was supported by the Natural Sciences and Engineering Research Council of Canada (NSERC) under a URF grant.

The author is with the Department of Electrical Engineering, University of Toronto, 10 King's College Road, SF1107, Toronto, ON M5S 1A4, Canada.

IEEE Log Number 9201547. fully characterize the interference we would have to fully specify this stochastic field.

Two fundamental processes drive the network self-interference component of the stochastic field: 1) the positions of network terminals and 2) the transmission characteristics of each terminal. Terminal transmissions are either due to external requests or to retransmissions required in the relaying of messages. In most research work, the external messages are assumed to arrive according to a Poisson process, if a continuous time model of the system is used, or according to a Bernoulli process for a discrete time model. The process describing the terminal positions is typically unknown and is beyond the control of the network designer. In many cases, the terminals are highly mobile and the set of terminal positions is time varying, and in other cases, we would like to obtain average performance for a class of random networks. In the case of bursty traffic, the set of interferers is not predictable for a given packet transmission. One way to handle all of these cases is to assume that the terminal positions are completely random and are distributed according to a Poisson point process in the plane. The Poisson process is a fundamental point process that is easy to handle analytically and its use leads to interference noise models that are more general than the Gaussion model and better suited to the network interference environment.

In this paper, we characterize the distribution of network self-interference for an idealized infinite network on the plane and obtain the probability of error as a function of the distance of a typical link for the spread spectrum schemes, direct sequence with binary phase shift keying (DS/BPSK), frequency hopping with $M$-ary frequency shift keying ( $\mathrm{FH} / \mathrm{MFSK}$, and frequency hopping with on-off keying (FH/OOK), where a sinusoidal tone is transmitted as the "on" symbol. The results obtained will be relevant to the understanding of ground radio packet networks with mobile terminals and bursty traffic, or to the understanding of dense networks whose terminals have a low transmission duty cycle.

\section{MODEL}

We assume a radio network whose terminals are distributed on the plane and we wish to determine the 
average performance of a link as a function of its distance. Many stochastic parameters need to be modeled in such a network; among them we have those related to the set of terminal positions, signaling waveforms, transmission times, uncertainty in terminal clocks, and signal propagation characteristics. All of these parameters will have some effect on the distribution of the interference at the receiver of interest. Perhaps the most important of these is the set of terminal positions. In our model the basic unit of time is the slot, which is equal to the packet transmission time. We assume that all terminals are synchronized at the slot level; no finer synchronization is assumed. With all of the random parameters that we will introduce to describe various system characteristics we assume independence from slot to slot. During a particular slot, we assume that the set of transmitting terminals (the set of interferers) forms a Poisson point process on the plane. Quantitatively, for a region $\boldsymbol{R}$ with area $A$ the number of transmitting terminals in the region has a Poisson distribution with parameter $\lambda A$, i.e.,

$$
P[k \text { in } R]=\frac{e^{-\lambda A}(\lambda A)^{k}}{k !},
$$

where $\lambda$ is the expected number of terminals per unit area.

We make the assumption that as a signal propagates its power decreases according to the function $g(r)=1 / r^{\gamma}$, where $r$ is the distance from the transmitter. We will generally work with the amplitude of a signal rather than the power, and define the amplitude loss function as

$$
a(r)=\frac{1}{r^{\gamma / 2}} .
$$

We are only interested in the behavior of the amplitude loss function with distance and do not account for any proportionality constant since it would scale all signals in the network by the same factor. This function is a far field approximation and suggests that the power of the signal becomes infinite as $r$ approaches zero. As a result in [5] the authors use the truncated function

$$
a_{t}(r)=\min \left(r^{-\gamma / 2}, s\right),
$$

for some $s>0$. However, in our case the use of (2) will result in the interference signal having a stable distribution, thus making the analysis tractable. We will give an example to indicate typical values of $s$ for which the above two functions give approximately equal results.

The interference at a point will depend on the form of the transmitted signals in the network. Examples of these are tones at various frequencies such as in a FH/MFSK system, and random looking signals such as in a direct sequence spread spectrum system [6]. We will assume the standard correlator receiver for DS/BPSK and the standard non-coherent receiver employing a bank of envelope detectors and a decision rule that selects the symbol corresponding to the maximum envelope in the case of FH/MFSK. For OOK, a threshold detector with optimum threshold is used. In the case of direct sequence, we assume random spreading codes. For FH/MFSK we assume that there are $Q$ orthogonal tones which are grouped into groups of $M$ tones, where $Q=q M$ and $q$ is the number of hopping slots. Note that for OOK $Q=q$. We denote the set of hopping frequencies as $\left\{f_{1}, f_{2}, \cdots, f_{q}\right\}$. One symbol is transmitted per hop and the hopping frequencies are random subject to the constraint that a sequence may not take the same value for two consecutive hops; i.e., if we denote the frequencies used at times $k$ and $k+1$ as $f^{k}$ and $f^{k+1}$ respectively, then $P\left[f^{k+1}=\right.$ $\left.f_{j} \mid f^{k}=f_{i}\right]=1 /(q-1)$ if $i \neq j$ and zero otherwise. This is the same random hopping model that is used for example in [7].

\section{DISTRIBUTION OF INTERFERENCE}

In the following, we find the probability density of the interference signal at a point in a Poisson field of interferers. For the signaling schemes that we are considering the set of terminals actually contributing to the interference can be obtained by splitting the original Poisson process and hence also forms a Poisson process with parameter which we denote as $\lambda_{t}$. In the case of DS/BPSK all terminals contribute to the interference hence $\lambda_{t}=\lambda$. For the moment we assume that the terminals are symbol synchronized. In the case of FH/MFSK, the set of terminals contributing to the interference at a particular tone forms a Poisson process with parameter $\lambda_{t}=\lambda / Q$, and in the case of $\mathrm{FH} / \mathrm{OOK}$, the parameter is $\lambda_{t}=\lambda /(2 Q)$, assuming that the transmitted symbols are equiprobable. The interference at the receiver, which we assume to be at the origin, is composed of a sum of components from each of the interferers. In the case of MFSK or OOK these components are sinusoids and in the case of DS /BPSK the components are random variables equal to the correlations of two DS signals. When conditioned on the transmission time offsets these random variables have a Gaussian probability density function in the case of large processing gain [8].

\section{A. General Procedure}

In some cases, we are interested in determining the probability of symbol error for some $M$-ary scheme. In other cases, we want to compute packet error probability assuming hard decision detection on each symbol, an error correcting block code, and dependent symbol errors; where the dependence arises due to the fact that the interferer configuration is constant over a packet.

We assume that the receiver consists of a set of correlators that produce a set of $n$ samples that we denote by the $n$-dimensional vector $\boldsymbol{Z}$. These samples are input to the detector/decoder. To compute the probability of error we need to determine the joint probability density function for the $n$-vector $Z$. As an example, for DS/BPSK the receiver consists of one correlator. To compute the probability of symbol error, we consider one output sample from the correlator, i.e., $n=1$. To compute the probability of packet error we take $n$ consecutive samples from 
the correlator, where $n$ is equal to the packet length. The resulting vector is the sum of a set of random vectors (referred to as influence functions) that account for the statistical dependence of bit errors in a packet. In the case of FH / MFSK, the receiver consists of a set of $2 M$ correlators. Thus, to compute the probability of symbol error we need the probability density function for the vector $Z$ whose components are the correlator outputs $(n=2 M)$. In the case of $\mathrm{FH} / \mathrm{OOK}$, the basic envelope detector receiver uses two correlators, hence to compute the symbol error probability we need to obtain the probability density function for a random vector with two components, i.e., $n=2$.

The received signal is given by

$$
z(t)=s(t)+\sum_{i} a\left(r_{i}\right) x_{i}(t)
$$

where $s(t)$ is the signal of interest and the sum is the interference signal. The summation is taken over all the interfering terminals, which form a Poisson process in the plane with parameter $\lambda_{t}$. The attenuation of the signals with distance has been explicitly included so that $x_{i}(t)$ is independent of the distance of the interferer to the receiver, $r_{i}$, and is proportional to the transmitted signal. In computing the various correlations, the receiver performs a set of inner products with the set of basis functions $\left\{\varphi_{k}(t)\right\}_{k=1}^{n}$ to obtain

$$
\boldsymbol{Z}=\boldsymbol{S}+\boldsymbol{Y},
$$

where $S$ is the signal component,

$$
\boldsymbol{Y}=\sum_{i} a\left(r_{i}\right) \boldsymbol{X}_{i},
$$

and the $k$ th component of $X_{i}$ is the correlation of $x_{i}(t)$ with the function of $\varphi_{k}(t)$. In other words, at the receiver the signal from the $i$ th interferer, $x_{i}(t)$ is converted (projected) to the $n$-dimensional vector $\boldsymbol{X}_{i}$. We assume that the random vectors $\boldsymbol{X}_{i}$ are independent and identically distributed, and that the distribution of $\boldsymbol{X}_{i}$ is independent of $r_{i}$. When referring to one of these random vectors generically we will drop the subscript and simply denote it as $\boldsymbol{X}$. The distribution of $\boldsymbol{X}$ will depend on the modulation that is being used, the carrier phase of the interferer with respect to that of the signal, and the symbol starting time relative to that of the signal. We assume that all second order moments of $\boldsymbol{X}$ exist and are infinite, and that $X$ has zero-mean. In this paper, we neglect background noise.

Our task is to find the probability density function of $\boldsymbol{Y}$. Towards this end we compute the characteristic function

$$
\phi_{Y}(\boldsymbol{\omega}) \triangleq e^{\psi(\boldsymbol{\omega})}=\boldsymbol{E}\left(\exp \left(j \boldsymbol{\omega}^{T} \boldsymbol{Y}\right)\right),
$$

where the superscript $T$ denotes the transpose, and $\psi(\cdot)$ has been defined as the logarithm of the characteristic function. To obtain $\phi(\cdot)$ we use a procedure that was used in [9], [10] in a packet radio context, and has since been traced back to Holtsmark who used it in 1919 to find the probability density of the electric field strength due to a random distribution of ions in a gas, and subsequently to Chandrasekhar [11] who used it to determine the probability density of the gravitational field of a random constellation of stars. A general framework for this type of problem is given in [12, pp. 31-48], where the random variable $u=a(r) X$ is called an influence function. In this paper, we refer to $\boldsymbol{X}$ as the influence function. To compute the characteristic function, the sum in (6) is first restricted to all terminals in a disk centered at the receiver and having radius $b, D_{b}$; then we let $b \rightarrow \infty$. We may calculate the previous expectation by conditioning on the number of terminals in $\boldsymbol{D}_{b}$. For a Poisson point process, given that there are $k$ terminals in a region, the positions are independent and have a uniform distribution. Thus, we have

$$
\phi_{Y}(\boldsymbol{\omega})=\lim _{b \rightarrow \infty} \sum_{k=0}^{\infty} P\left[k \text { in } \boldsymbol{D}_{b}\right]\left(\boldsymbol{E}\left(\exp \left(j a(r) \boldsymbol{\omega}^{T} \boldsymbol{X}\right)\right)\right)^{k},
$$

where the expectation is over the random variables $\boldsymbol{X}$ and $r$ corresponding to the contribution to the total interference from a generic interferer. Now, using (1) we may compute the log-characteristic function as

$$
\begin{array}{r}
\psi(\boldsymbol{\omega})=\lim _{b \rightarrow \infty} \lambda_{t} \pi b^{2}\left(\int_{0}^{b} \boldsymbol{E}\left(\exp \left(j a(r) \boldsymbol{\omega}^{T} \boldsymbol{X}\right) \mid R=r\right)\right. \\
\left.\cdot f_{R}(r) d r-1\right),
\end{array}
$$

where the expectation is over the random vector $\boldsymbol{X}$ and $f_{R}(\cdot)$ is the probability density of the distance $r$. The probability density of the distance to the origin, $r$, of a point whose position has the uniform distribution in a disk of radius $b$ is

$$
f(r)= \begin{cases}\frac{2 r}{b^{2}}, & r \leq b, \\ 0, & \text { elsewhere. }\end{cases}
$$

Thus, (9) becomes

$$
\psi(\boldsymbol{\omega})=\lim _{b \rightarrow \infty} \lambda_{t} \pi b^{2}\left(\int_{0}^{b} \frac{2 r}{b^{2}} \Phi(a(r) \boldsymbol{\omega}) d r-1\right),
$$

where $\Phi(\cdot)$ is the characteristic function of $\boldsymbol{X}$. Integrating the above by parts, letting $b \rightarrow \infty$, and noting that $\lim _{b \rightarrow \infty} b^{2}(\Phi(a(b) \omega)-1)=0$, we obtain ${ }^{1}$

$$
\begin{aligned}
\psi(\boldsymbol{\omega}) & =-\lambda_{t} \pi \int_{0}^{\infty} r^{2} \frac{d \Phi(a(r) \omega)}{d r} d r \\
& =\lambda_{t} \pi \int_{0}^{\infty} \frac{d \Phi(t \omega)}{d t} t^{-\alpha} d t,
\end{aligned}
$$

where $\alpha=4 / \gamma$. At this point we impose the restriction that the random vector $\boldsymbol{X}$ has a spherically symmetric probability density function. The characteristic function is

\footnotetext{
${ }^{1}$ This limit is equivalent to $\lim _{x \rightarrow 0}\left[\Phi_{0}\left(x^{\gamma / 2}\|\boldsymbol{\omega}\|\right)-1\right] / x^{2}$. The result follows from the application of L'Hopital's rule and the assumption that $\boldsymbol{X}$ has zero-mean and finite second-order moments.
} 
then also spherically symmetric and we may write it as $\Phi(\boldsymbol{\omega})=\Phi_{0}(\|\boldsymbol{\omega}\|)$, where $\|\cdot\|$ is the Euclidean norm. As a result, we may simply (12) to

$$
\psi(\boldsymbol{\omega})=\lambda_{t} \pi\|\boldsymbol{\omega}\|^{\alpha} \int_{0}^{\infty} \frac{\Phi_{0}^{\prime}(x)}{x^{\alpha}} d x .
$$

We note that the integral in (13) is independent of $\boldsymbol{\omega}$. Thus, the effect of the distribution of $\boldsymbol{X}$ is merely to scale the total interference, and

$$
\psi(\boldsymbol{\omega})=-\sigma\|\boldsymbol{\omega}\|^{\alpha},
$$

where $\sigma=\beta \lambda_{t}$ and

$$
\beta=-\pi \int_{0}^{x} \frac{\Phi_{0}^{\prime}(x)}{x^{\alpha}} d x .
$$

Equation (14) is the log-characteristic function for the spherically symmetric stable distribution of exponent $\alpha$.

If instead of (2) we use (3) as the amplitude loss function then the log-characteristic function is the same as before except that $\beta$ is dependent on $\boldsymbol{\omega}$ as follows:

$$
\beta(\omega)=-\pi \int_{0}^{\|\omega\| s^{-2 / \alpha}} \frac{\Phi_{0}^{\prime}(x)}{x^{\alpha}} d x .
$$

For a fixed $s$ the difference between the two log-characteristic functions approaches zero rapidly as $\|\boldsymbol{\omega}\| \rightarrow \infty$. For small $\|\boldsymbol{\omega}\|$ the difference approaches zero as $s \rightarrow 0$. As an example let $\lambda_{t}=1$ (this amounts to choosing a scale for $r), \alpha=1$, and $X=(\cos \theta, \sin \theta)$, then the two $\log$-characteristic functions are practically indistinguishable if $s<0.1$. For $s=0.1$ the expected number of interferers in the region where $r \leq s$ (the "flat region") is equal to $\pi / 100$.

For the integral in (13) to converge at 0 , excluding the trivial case of zero second-order moments of $\boldsymbol{X}$ we must have $\alpha<2$; also since $\Phi_{0}^{\prime}(0)=0$ and $\Phi_{0}^{\prime \prime}(0)$ exists the convergence at 0 holds for all $0<\alpha<2$. Convergence at the upper limit holds for $\alpha>0$. The symmetric stable distributions are characterized by the parameter $0<\alpha \leq$ 2. Thus, depending on $\gamma$ we obtain any of the stable distributions except the Gaussian distribution $(\alpha=2)$. The corresponding restriction on $\gamma$ is $\gamma>2$. Thus the case of free space propagation cannot be included in this framework.

We are mainly interested in the case of ground wave propagation where a commonly assumed value, resulting from a two-ray propagation model [13, pp. 81-83], is $\gamma=4$. For such a value we have $\alpha=1$. The resulting distribution is the $n$-variate Cauchy distribution with parameter $\sigma$ whose density is given by

$$
f_{Y}(y)=\frac{\Gamma[(n+1) / 2]}{\sigma^{n} \pi^{(n+1) / 2}}\left(1+\frac{1}{\sigma^{2}}\|\boldsymbol{y}\|^{2}\right)^{-(n+1) / 2} .
$$

In Table I, we give $\beta$ values for five different univariate distributions. The $\beta$ value is a measure of the spread of the distribution (similar to the standard deviation). The last row contains the compound Gaussian distribution
TABLE I

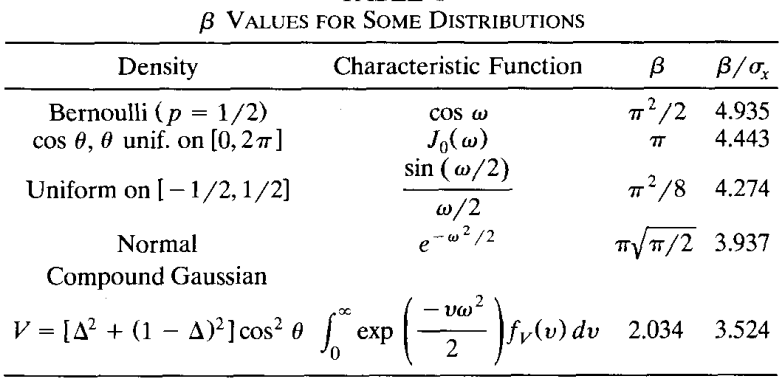

with conditional variance $V$. The random variables $\Delta, \theta$ have the uniform distribution on $[0,1]$ and $[0,2 \pi]$, respectively. This compound Gaussian random variable occurs in the modeling of the interference in a direct sequence spread spectrum multiple access system with a large processing gain where the spreading code chips are assumed to be non-synchronized, and the chips have a rectangular pulse shape, $\Pi(t)=1$ if $|t|<1 / 2$, 0 otherwise.

\section{B. Specific Cases}

The distributions in Table I may be applicable for different signaling schemes. As an example for a direct sequence spread spectrum system with a large processing gain and chip synchronization among all the users, we may model the noise at the detector due to each interferer as a Gaussian random variable. Thus, if we are considering one symbol $(n=1)$, the resulting noise distribution, assuming a $1 / r^{4}$ power loss law, is Cauchy with parameter $\sigma=\beta \lambda_{t}$ where $\beta=3.937 \sigma_{x}$, and $\sigma_{x}$ is the standard deviation of $X$.

In the case of frequency hopping, the interference consists of sinusoidal tones. Thus, to compute the probability density of the envelope we need to obtain the joint probability density for the in-phase and quadrature correlator outputs. In this case, $\boldsymbol{X}=(\cos \theta, \sin \theta)$ where $\theta$ is assumed to be uniformly distributed in $[0,2 \pi]$. This density is spherically symmetric (in two dimensions) and gives the value $\beta=\pi$. Thus, the probability density of $\boldsymbol{Y}$ is the bivariate Cauchy with parameter $\sigma=\pi \lambda_{t}$, i.e.,

$$
f_{Y}(y)=\frac{\lambda_{t} / 2}{\left(\left(\lambda_{t} \pi\right)^{2}+\|y\|^{2}\right)^{3 / 2}} .
$$

For the performance of an envelope detector, we are also interested in the distribution of the envelope of the signal

$$
W=\|\boldsymbol{Y}\| .
$$

The probability density of the envelope is

$$
f_{W}(w)= \begin{cases}\frac{\lambda_{t} \pi w}{\left(\left(\lambda_{t} \pi\right)^{2}+w^{2}\right)^{3 / 2}}, & \text { if } w \geq 0, \\ 0, & \text { otherwise. }\end{cases}
$$

We may also consider the case of DS/BPSK where no assumption on the chip synchronization is made. In the 
case of a rectangular pulse shape and $n=1$, the resulting probability density for $\boldsymbol{X}$ is the compound Gaussian density whose $\beta$ value is the last entry in Table I. We note that this $\beta$ value is smaller than that for the Gaussian law, hence the lack of chip synchronization tends to result in less interference.

In the case of Gaussian or compound Gaussian probability, laws for $\boldsymbol{X}$ the $\beta$ values are not dependent on $n$ and the previous examples easily generalize to the multivariate case, or the case where the joint probability density over all the symbols of a packet is required. In fact we may consider the general $n$-variate symmetric compound Gaussian density which may be viewed as an $n$-variate Gaussian with random variance $V$ having probability density function $f_{V}(v)$. We assume that the random "variance" is normalized so that $\boldsymbol{E}(V)=1$. The characteristic function is then

$$
\Phi(\boldsymbol{\omega})=\int_{0}^{\infty}-D\|\omega\|^{2} / 2 f_{V}(v) d v .
$$

Note that the $n$-variate normal distribution is a special case of the $n$-variate compound Gaussian distribution for which $f_{V}(v)=\delta(v-1)$. We may obtain the $\beta$ parameter for the general compound Gaussian distribution as follows:

$$
\beta=\beta_{N} \int_{0}^{\infty} \sqrt{v} f_{V}(v) d v
$$

where $\beta_{N}$ is the $\beta$ parameter for the $n$-variate normal distribution. From (22) we see that the $\beta$ parameter is reduced by a factor equal to $\boldsymbol{E}(\sqrt{V})$. Under the constraint $\boldsymbol{E}(V)=1$ this factor attains the maximum value of 1 for $f_{V}(v)=\delta(v-1)$ and is less than 1 for all other distributions. On the other hand, there is no nonzero lower bound for $\boldsymbol{E}(\sqrt{V})$. If we choose the distribution $f_{V}(v)=$ $(1-1 / n) \delta(v)+(1 / n) \delta(v-n)$, then $\boldsymbol{E}(\sqrt{V}) \rightarrow 0$ as $n$ $\rightarrow \infty$.

\section{Nonsynchronized Frequency Hopping}

Up to now we assumed that in the case of frequency hopping the hops of the different users were synchronized. We now drop this assumption. With nonsynchronized hopping it is not possible to keep the different tones strictly orthogonal as in the case of synchronized hopping. An interferer can interfere with a signal tone (a frequency slot) in one of two ways. When the same tone is transmitted we call it a hit, when a different tone is transmitted we call it a splash. In both cases the interference can be constructive or destructive depending on the sign of the interference component at the detector output. The strength of a splash depends mainly on the frequency separation between the two tones and on the pulse shape. Our notion of a hit is the same as in previous analysis of frequency hopping multiple access, however we treat its effect more accurately since we work with the actual signal levels at the detector output. Due to intractability, the effect of splashes has been neglected in previous analysis. In our case, the effect could be included more easily due to our framework of a Poisson field of interferers. However, in many cases the result would be a slight increase in the $\beta$ parameter; hence we also neglect it so as not to unnecessarily obscure the analysis.

We assume that the hopping time is normalized to unity and the hopping sequence of the $i$ th interferer is out of phase with the sequence of the signal of interest by the amount $\Delta_{i}$, where $\Delta_{i}$ is a random variable with uniform distribution on the interval $[0,1]$. The probability that an interfering tone overlaps fully the signal of interest is zero. If the time interval of the $j$ th hop of the signal is $[j$, $j+1]$ then we say that the $i$ th interferer hits the signal on the left if it uses the same frequency slot as the signal and its hop interval is $\left[j-\Delta_{i}, j+1-\Delta_{i}\right]$; the interferer hits the signal on the right if it uses the same frequency slot as the signal and its $j$ th hop interval is $\left[j+1-\Delta_{i}, j+2-\right.$ $\Delta_{i}$ ]. If the $i$ th interferer hits the signal of interest in a given slot then it will hit on the left, or on the right, but not both due to the fact that the hopping frequency must change at every hop. Let $H_{l}$ and $H_{r}$ be the indicator functions of the events corresponding to hits by the $i$ th interferer on the left and right respectively. The random variables $\left(H_{l}, H_{r}\right)$ have the joint probabilities $P\left[H_{l}=1\right.$, $\left.H_{r}=0\right]=P\left[H_{l}=0, H_{r}=1\right]=1 / Q$ and $P\left[H_{l}=1, H_{r}=\right.$ 1) $]=0$. Dropping the interferer index $i$, the influence function then becomes ${ }^{2}$

$$
\boldsymbol{X}=U(\cos \theta, \sin \theta),
$$

where $U=H_{l} R_{m}(1-\Delta)+H_{r} R_{m}(\Delta)$, and where $R_{m}(\tau)$ $=\int_{-\infty}^{\infty} m(t) m(t+\tau) d t$ is the autocorrelation function of the baseband pulse shape function, $m(t)$.

We note that $\boldsymbol{X}$ has a spherically symmetric density. The $\beta$ factor may be computed as before. For $\alpha=1$ the $\beta$ factor is given by $\beta=\pi \int_{0}^{1}\left(R_{m}(\tau)+R_{m}(1-\tau)\right) d \tau$. Thus, if $m(t)=\Pi(t)$ then $\beta=\pi$, and if $m(t)$ is a cosine pulse (i.e., $m(t)=\sqrt{2} \cos (\pi t)$ for $|t|<1 / 2,0$ otherwise) then $\beta=8 / \pi$.

We note that for the rectangular pulse there is no change in the $\beta$ factor when compared to the case of synchronized hopping. For other pulse shaping functions the $\beta$ factor is actually smaller with nonsynchronized hopping. We may, therefore, draw the important conclusion that, if the effect of splashes is not significant, then nonsynchronized hopping will not degrade the performance and may actually increase it, i.e., the probability of symbol error may even be lower. In fact, the lack of hopping time synchronization does not create extra dependencies in symbol errors. In past analysis of the multiple access capability of frequency hopping schemes, a combinatoric approach is taken to calculate the probability of symbol error due to hits. In this model, a hit is assumed to cause an error with a given probability regardless of its degree of overlap. The results obtained lead to

\footnotetext{
${ }^{2}$ The waveform at the receiver is projected onto the two basis functions $\varphi_{1}(t)=\sqrt{2} m(t) \cos (\omega t)$ and $\varphi_{2}(t)=\sqrt{2} m(t) \sin (\omega t)$, where $\omega$ is the frequency of the corresponding tone.
} 
the conclusion that nonsynchronized hopping causes a significant degradation in performance if the number of symbols per hop is small (e.g., see (24) in [5]). We note that in our case, under the assumption of a Poisson field of interferers, this degradation is not present, and depending on the tone separation and pulse shape (i.e., effect of splashes) nonsynchronized hopping may result in improved performance over synchronized hopping.

\section{Symbol ERror Probability vs. Distance}

In this section, we use the preceding results for the distribution of the total interference at a receiver to determine the probability of symbol error versus the link distance for the schemes FH/OOK, FH/MFSK, and DS/BPSK. Let $\mathrm{R}$ be the link distance. The quality of the link will depend on the distance and also on the expected density of terminals, $\lambda$. We define the parameter $N$, which incorporates both of these, as

$$
N \triangleq \lambda \pi \mathrm{R}^{2} \text {. }
$$

Our results will be expressed in terms of the dimensionless parameter, $N$. The parameter $N$ is equal to the expected number of interferers that are closer to the receiver than the transmitter.

\section{A. $\mathrm{FH} / \mathrm{OOK}$}

Denote the symbol transmitted when the transmitter is on or off by " 1 " and " 0 ", respectively. We assume that the receiver processes the received waveform to obtain the two-dimensional random vector $Z$ as in (5). To derive the minimum probability of error receiver we assume equiprobable binary signaling and let $\boldsymbol{H}_{0}$ and $\boldsymbol{H}_{1}$ be the hypothesis that a " 0 " and a " 1 " is transmitted respectively. Given the linear processing to obtain $Z$ the optimum receiver calculates the following maximum likelihood ratio

$$
\Lambda(Z)=\frac{f_{Z \mid H_{0}}(Z)}{f_{Z \mid H_{1}}(Z)}
$$

and decides on a " 0 " or " 1 " if $\Lambda$ is greater or less than 1 , respectively.

The conditional density $f_{Z H_{0}}(\cdot)$ is equal to $f_{Y}(\cdot)$ in (18). Assuming a $1 / r^{4}$ power loss law, and letting $Z=\left(Z_{1}\right.$, $Z_{2}$ ), under the hypothesis $\boldsymbol{H}_{1}$, the random variable $Z_{1}$ will have a signal component $(\cos \theta) / R^{2}$ and $Z_{2}$ will have a signal component $(\sin \theta) / R^{2}$, where $\theta$ is the phase angle of the signal, and is uniformly distributed on $[0,2 \pi]$. Thus,

$$
\begin{aligned}
f_{Z \mid H_{1}}\left(z_{1}, z_{2}\right)=\frac{1}{2 \pi} \int_{0}^{2 \pi} f_{Z \mid H_{0}} & \\
& \quad\left(z_{1}-\frac{\cos \theta}{R^{2}}, z_{2}-\frac{\sin \theta}{R^{2}}\right) d \theta .
\end{aligned}
$$

To simplify this expression let $\rho=\|Z\| / \sigma$ and $\mu_{O}=$
$1 /\left(\lambda_{t} \pi R^{2}\right)$, or in terms of system parameters, let

$$
\mu_{O}=\frac{2 Q}{N} \text {. }
$$

After some manipulation we can show that the likelihood ratio (25) becomes a function of the single variable $\rho$ :

$$
\Lambda(\rho)=\frac{\frac{\pi}{2}\left(1+\left(\rho-\mu_{O}\right)^{2}\right) \sqrt{1+\left(\rho+\mu_{O}\right)^{2}}}{\left[1+\rho^{2}\right]^{3 / 2} E_{0}\left(\sqrt{\frac{4 \rho \mu_{o}}{1+\left(\rho+\mu_{O}\right)^{2}}}\right)}
$$

where $E_{0}(\cdot)$ is the complete elliptic integral of the second kind.

$$
E_{0}(k)=\int_{0}^{\pi / 2} \sqrt{1-k^{2} \sin ^{2} t} d t .
$$

For the minimum probability of error receiver, we need the threshold $\rho^{*}$ where $\Lambda\left(\rho^{*}\right)=1$. By numerical analysis we obtain the approximate relationship $\rho^{*} \approx 0.66 \mu_{O}$ that holds for $\mu_{O}>4$.

The average probability of symbol error is computed as

$$
p_{e}=\frac{1}{2}(P[\text { Error } \mid 0]+P[\text { Error } \mid 1]) \text {. }
$$

The conditional probability of error given " 0 " can be obtained in closed form by using (20) as

$$
P[\text { Error } \mid 0]=\frac{1}{\sqrt{1+\rho^{* 2}}} \approx \frac{1}{\sqrt{1+0.435 \mu_{O}^{2}}} .
$$

The conditional probability $P[$ Error $/ 1]$ was obtained numerically. For $\mu_{O}>10$, we have the following approximations:

$$
P[\text { Error } \mid 0] \approx \frac{1.5}{\mu_{O}}, \quad P[\text { Error } \mid 1] \approx \frac{0.41}{\mu_{O}},
$$

and

$$
p_{e} \approx \frac{0.96}{\mu_{O}}
$$

\section{B. $F H / M F S K$}

We consider the case of $M$-ary frequency shift keying with synchronized hopping times. It can be shown, as previously, that the nonsynchronized case presents no major difficulties and leads to the same probability of symbol error. The receiver is a noncoherent receiver consisting of $M$ envelope detectors and a decision rule that chooses the symbol corresponding to the envelope of maximum value. Let the outputs of the envelope detectors be $W_{0}, W_{1}, \cdots, W_{M-1}$. We can show that the $W_{i}$ 's are independent random variables. We may do this by using the influence function

$\boldsymbol{X}=\left(\delta_{0} \cos \theta_{0}, \delta_{0} \sin \theta_{0} ; \delta_{1} \cos \theta_{1}, \delta_{1} \sin \theta_{1} ; \cdots ;\right.$

$$
\left.\delta_{M-1} \cos \theta_{M-1}, \delta_{M-1} \sin \theta_{M-1}\right),
$$


where the $\theta_{i}$ 's are independent and identically distributed (i.i.d.) random variables with uniform distribution on $[0$, $2 \pi$ ]. The $\delta_{i}$ 's are random variables with values in the set $\{0,1\}$ and distribution given by $P\left(\delta_{0}, \cdots, \delta_{M-1}\right)=1 / M$ if exactly one of the arguments equals one, and zero otherwise.

The random variables $W_{j}$ are obtained as follows:

$$
W_{j}=\left\|\boldsymbol{Z}^{(j)}\right\|, \quad j=0,1, \cdots, M-1
$$

where $Z^{(j)}=\left(Z_{1}^{(j)}, Z_{2}^{(j)}\right), Z_{1}^{(j)}$, and $Z_{2}^{(j)}$ being the in-phase and quadrature integrator outputs for the $j$ th envelope detector. We assume that the signal tone is contained in the 0 th-frequency slot. For $\alpha=1$, the probability density function for $\boldsymbol{Z}^{(j)}$ is then a bivariate Cauchy given by (18). The probability density function for the $W_{j}, j=1, \cdots, M-$ 1 , is given by (20) with the appropriate $\lambda_{t}$. To compute the probability of symbol error it is advantageous to view the standard $n$-variate Cauchy density (i.e., (17) with $\sigma=1$ ) as the following compound Gaussian density with random variance $V$ :

$$
f_{Y}(y)=\int_{0}^{\infty} \frac{1}{(2 \pi v)^{n / 2}} \exp \left(\frac{-\|y\|^{2}}{2 v^{2}}\right) f_{V}(v) d v
$$

where $f_{V}(\cdot)$ is the inverse Gaussian density given by

$$
f_{V}(v)=\frac{1}{\sqrt{2 \pi}} v^{-3 / 2} \exp (-1 /(2 v))
$$

We let $V_{j}$ be the conditional variance for $Z^{(j)}, j=0, \cdots, M$ - 1. Conditioned on the random variable $V_{j}$, the random variables $W_{j}(j \neq 0)$ have Rayleigh distributions, and conditioned on $V_{0}$ the random variable $W_{0}$ has a Rician distribution.

$$
\begin{array}{r}
f_{W_{j}}(r)=\int_{0}^{\infty} \frac{r}{v} \exp \left(-\frac{r^{2}}{2 v}\right) f_{V}(v) d v, \\
j=1, \cdots, M-1, \\
f_{W_{0}}(r)=\int_{0}^{\infty} \frac{r}{v} \exp \left(-\frac{1}{2 v}\left(r^{2}+\mu_{M}^{2}\right)\right) \\
I_{0}\left(\frac{r \mu_{M}}{v}\right) f_{V}(v) d v,
\end{array}
$$

where $r$ and $\mu_{M}$ are normalized values as in the previous section. Note that in this case, since each terminal always transmits a tone, we have

$$
\mu_{M}=\frac{Q}{N}
$$

We thus obtain the probability of symbol error as follows:

$$
\begin{aligned}
p_{e}= & \boldsymbol{E}\left(P\left[\text { Error } \mid V_{0}, V_{1}, \cdots, V_{M-1}\right]\right) \\
= & 1-\boldsymbol{E}\left(P \left[W_{1}<W_{0}, W_{2}<W_{0}, \cdots,\right.\right. \\
& \left.\left.W_{M-1}<W_{0} \mid \boldsymbol{V}\right]\right) \\
= & E\left(\int_{0}^{\infty}\left[1-\prod_{j=1}^{M-1}\left(1-\exp \left(-\frac{r}{2 V_{j}}\right)\right)\right] f_{W_{0}}(r) d r\right),
\end{aligned}
$$

where $\boldsymbol{E}(\cdot)$ is an expectation over the random variance vector

$$
V=\left(V_{0}, V_{1}, \cdots, V_{M-1}\right)
$$

This computation is straightforward once we realize that the conditional variances $V_{j}$ are i.i.d. random variables. We obtain

$$
p_{e}=\sum_{k=1}^{M-1}(-1)^{k+1}\left(\begin{array}{c}
M-1 \\
k
\end{array}\right) \rho_{k},
$$

where

$$
\rho_{k}=E\left(\int_{0}^{\infty} \prod_{j=1}^{k} \exp \left(\frac{-r^{2}}{2 V_{j}}\right) f_{W_{0}}(r) d r\right)
$$

and the $V_{j}$ 's are a subset of the initial set of $M$ variances that has been reindexed. The expectation is over the random variables $V_{1}, \cdots, V_{k}$.

To compute the $\rho_{k}$ 's we note that the random variable $\xi=1 / V$ has the density

$$
f_{\xi}(t)=\frac{1}{\sqrt{2 \pi t}} \exp (-t / 2)
$$

The sum $\zeta=\sum_{j=1}^{M-1} 1 / V_{j}$ has a Gamma density. To perform the computation of $\rho_{k}$ we first take the expectation with respect to $V_{0}$ followed by the expectation with respect to $\zeta$ and finally integrate with respect to $r$. After some substitutions we obtain

$\rho_{k}=\frac{1}{\sqrt{\pi}} \frac{\Gamma[(k+1) / 2]}{\Gamma(k / 2)} \int_{0}^{\pi} \frac{\sin ^{2} \frac{\phi}{2} \cos ^{k-1} \frac{\phi}{2}}{\left[1+\frac{\mu_{M}^{2}}{4} \sin ^{2} \phi\right]^{(k+1) / 2}} d \phi$

This integral may be computed directly for small values 
of $k$ to obtain for example

$$
\begin{array}{ll}
\rho_{1}=\frac{1}{\sqrt{4+\mu_{M}^{2}}}, & \\
\rho_{2}=\frac{1}{\mu_{M}^{2}}\left[\rho^{2}+\frac{1}{2} \sqrt{\rho(1-\rho)^{2}} F[a, b]\right] & -\frac{2 \rho^{3 / 2}}{\mu_{M}^{3}} E[a, b], \\
\rho_{3}=\frac{2}{\left(4+\mu_{M}^{2}\right)^{3 / 2}},
\end{array}
$$

where $\rho=\mu_{M} / \sqrt{4+\mu_{M}^{2}}, a=\sin ^{-1} \sqrt{(1-\rho) /(1+\rho)}$, and $b=\sqrt{(1+\rho) / 2}$. The functions $F(\cdot, \cdot)$ and $E(\cdot, \cdot)$ are defined as

$$
\begin{aligned}
& F(x, k)=\int_{x}^{\pi / 2} \frac{1}{\sqrt{1-k^{2} \sin ^{2} \phi}} d \phi, \\
& E(x, k)=\int_{x}^{\pi / 2} \sqrt{1-k^{2} \sin ^{2} \phi} d \phi .
\end{aligned}
$$

These are complements of elliptic integrals of the first and second kind, respectively.

In the previous examples, for large $\mu_{M}$ the parameter $\rho$ approaches unity. In the expression for $\rho_{2}$ the term containing the $F$ function approaches zero as $\mu_{M} \rightarrow \infty$. In general, for large $\mu_{M}, \rho_{k} \sim 1 / \mu_{M}^{k}$. As a result, we have

$$
p_{e} \sim \frac{M-1}{\mu_{M}}, \quad \mu_{M} \gg 1 .
$$

\section{C. $D S / B P S K$}

We now consider DS/BPSK. We assume random spreading codes with $L$ chips per data symbol; $L$ is the processing gain. We assume a rectangular chip shape function $\Pi(t)$. Let $\Delta$ and $\theta$ be the relative chip phase between the spreading code and carrier of the signal and a generic interferer respectively. We assume that these have uniform distributions in $[0,1]$ and $[0,2 \pi]$, respectively. Conditioned on $\Delta$ and $\theta$, for large $L$, the interferer contributes an influence function $X$ at the detector that has a Gaussian distribution [8] with variance

$$
V_{1}=L\left[\Delta^{2}+(1-\Delta)^{2}\right] \cos ^{2} \theta
$$

The unconditional probability density is a compound Gaussian. Referring to the last entry of Table I, writing $\beta_{d s}=2.035$, and noting that the $\beta$ parameter is proportional to the standard deviation, we have in this case $\beta=\beta_{d s} \sqrt{L}$. The resulting interference due to all of the interferers is Cauchy distributed with parameter $\sigma=\lambda_{t} \beta_{d s}$ $\sqrt{L}$. The probability density is

$$
f_{Y}(y)=\frac{\lambda_{t} \beta_{d s} \sqrt{L}}{\pi\left(\lambda_{t}^{2} \beta_{d s}^{2} L+y^{2}\right)} .
$$

As a result of symmetry the detector threshold which minimizes the probability of error is zero, and the probability of error is equal to the conditional probability of error (given " 0 " or " 1 "). Assuming that the link distance is $R$, the received signal value is $L / R^{2}$. The probability of error is then

$$
p_{e}=P\left[Y>\frac{L}{R^{2}}\right],
$$

where $Y$ is the interference and has a probability density given by (51).

Solving (52), we obtain

$$
p_{e}=\frac{1}{2}-\frac{1}{\pi} \tan ^{-1} \mu_{D},
$$

where

$$
\mu_{D}=\frac{\pi \sqrt{L}}{N \beta_{d s}} .
$$

For $x \geqslant 1, \tan ^{-1}(x) \approx \pi / 2-1 / x$; hence (53) may be approximated by $p_{e} \approx 1 /\left(\pi \mu_{D}\right)$.

\section{CODEd PERFormance}

We assume a packet consisting of $n$ symbols and a $t$-error correcting block code. In calculating packet error probability dependencies between error events must be taken into account. For the DS/BPSK, we have used a vector influence function to obtain the probability density for the interference over all of the symbols in the packet, hence these dependencies have been taken into account. Let $Y$ be the interference noise vector over the $n$ symbols of the packet. This noise vector has a probability density given by (17), where $\beta=\beta_{d s} \sqrt{L}$ as in (51) and $\sigma=$ $\lambda_{t} \beta_{d s} \sqrt{L}$. Let $U=Y / \sigma$. The random vector $U$ has the standard $n$-variate Cauchy distribution. Let $Y_{i}$ and $U_{i}$ be components of the previous noise vectors, and let $\mu^{\prime}$ be the threshold on $Y_{i}$ for which a negative valued symbol is detected in error when the noise exceeds the threshold. From (52), we have $\mu^{\prime}=L / R^{2}$, hence the threshold on $U_{i}$ is $\mu^{\prime} /\left(\lambda_{t} \beta_{d s} \sqrt{L}\right)$ that reduces to $\mu_{D}$ as given by $(54)$. Now, as in (36), the standard Cauchy density may be written as a compound Gaussian. Thus, the block error probability may be obtained by conditioning on the "variance" as

$$
\begin{aligned}
P_{e}=\int_{0}^{\infty} \sum_{i=t+1}^{n}\left(\begin{array}{c}
n \\
i
\end{array}\right)(1 & \left.-\frac{1}{2} \operatorname{erfc}\left(\frac{\mu_{D}}{\sqrt{2 v}}\right)\right)^{n-i} \\
& \cdot\left(\frac{1}{2} \operatorname{erfc}\left(\frac{\mu_{D}}{\sqrt{2 v}}\right)\right)^{i} f_{V}(v) d v,
\end{aligned}
$$

where $f_{V}(\cdot)$ is given by (37) and where $\operatorname{erfc}(x)=$ $(2 / \sqrt{\pi}) \int_{x}^{\infty} \exp \left(-t^{2}\right) d t$.

We would like to obtain an expression for the probability of packet error for larger packet sizes $n$ under the assumption that an error-correcting code of error-correcting capability $t$ is used. Such a result is difficult to obtain in closed form. We, therefore, find limiting values 
as $n \rightarrow \infty$. Let $n \rightarrow \infty$ and $t \rightarrow \infty$ in such a way that $\epsilon=t / n$ is constant. The following limit is readily available from (55):

$$
P_{e x}=\operatorname{erf}\left(\frac{1}{\mu_{D}} \operatorname{erfc}^{-1}(2 \epsilon)\right)
$$

where erfc $(x)=1-\operatorname{erfc}(x)$. Note that for large $\mu_{D}$ $\left(\mu_{D} \geqslant \operatorname{erfc}^{-1}(2 \epsilon)\right)$ we have $P_{e x} \approx\left(1 / \mu_{D}\right) \operatorname{erfc}^{-1}(2 \epsilon)$ Thus, for a large $n$ and a fixed amount of coding, the probability of packet error is inversely proportional to $\mu_{D}$ for large values of $\mu_{D}$. We note that this result is different than that of a memoryless BSC channel where the limiting probability is either 0 or 1. Equation (56) has been plotted in Fig. 1 as a function of the threshold parameter $\mu_{D}$ for three different values of the parameter $\epsilon$. The dependence between symbol errors due to the Cauchy distribution results in a smooth limiting probability of error curve; if the symbol errors were independent the curve would be a step function.

In the case of frequency hopping, it is more difficult to account for dependencies between errors since we have not used a vector influence function, encompassing multiple symbols, in obtaining the distribution of the interference. In the case, where it is possible for an interferer to hit more than one symbol in a packet, symbol errors will generally be dependent. The degree of correlation between errors depends on the parameters $N$ and $Q$. For large $Q$ and small $N$, the correlation between errors will be weak. However, if the hopping sequences are specifically designed so that there is at most one hit between any two sequences during a packet transmission, then symbol errors will be independent and the packet error probability for a block code can easily be calculated from the symbol error probabilities already obtained. This is the case for both $\mathrm{FH} / \mathrm{OOK}$ and FH/MFSK.

We would like to compare the various signaling schemes using a common criteria. Since the different signaling schemes use different alphabet sizes, the packet error probability is not a good measure since different schemes have different information transmission rates. Instead we use the channel capacity. Under the assumption that symbol errors are independent, the capacity for the $\mathrm{FH} / \mathrm{MFSK}$ schemes is readily obtained as

$$
C_{M}=\left(1-p_{e}\right) \log _{2} M+p_{e} \log _{2} \frac{M}{M-1}-H\left(p_{e}\right),
$$

where $H(x)=-x \log _{2} x-(1-x) \log _{2}(1-x)$ is the binary entropy function. In the case of $\mathrm{FH} / \mathrm{OOK}$, the capacity is a bit more difficult to compute since the channel is not symmetric and the receiver that we have considered is that which minimizes the probability of error. Under the assumption of equally likely channel input symbols, the mutual information is a lower bound for the capacity given as

$$
C_{O}=H\left(\frac{1}{2}-\frac{1}{2}\left(p_{0}-p_{1}\right)\right)-\frac{1}{2}\left(H\left(p_{0}\right)+H\left(p_{1}\right)\right),
$$

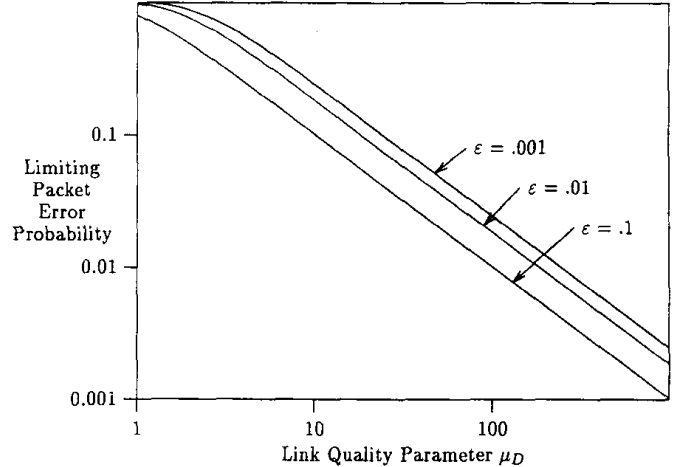

Fig. 1. Limiting packet error probability for codes with different error correcting capability.

where $p_{0}$ and $p_{1}$ are the conditional probabilities of error.

Lastly we would like a comparison with the DS/BPSK scheme. The capacity for DS/BPSK may be computed as the expectation $E\left(1-H\left(1 / 2 \operatorname{erfc}\left(\mu_{D} / \sqrt{2 V}\right)\right)\right)$ over the random variable $V$, which has the density given in (37). The result is

$$
C_{D}=1-\frac{2}{\sqrt{\pi}} \int_{0}^{\infty} H\left(\frac{1}{2} \operatorname{erfc}\left(\mu_{D} t\right)\right) \exp \left(-t^{2}\right) d t .
$$

The performance of the various signaling schemes is determined by the link quality parameters given by (27), (40), and (54). In order to compare these schemes, we need to express these parameters in terms of a common parameter. We define the expected number of interferers closer to the receiver than the transmitter, per frequency slot, as $G=N / Q$. Thus, $\mu_{M}=G^{-1}$ and $\mu_{O}=2 G^{-1}$. Now, to express $\mu_{D}$ in terms of $G$, we need to relate the bandwidth parameters $Q$ and $L$ for the frequency hopping and direct sequence schemes. Assuming a constant system bandwidth these parameters are both proportional to the bandwidth. Thus, we write $L=\kappa Q$ and we have

$$
\mu_{D}=\frac{\pi}{\beta_{d s}} \sqrt{\frac{\kappa}{Q}} G^{-1} \text {. }
$$

Let the tone spacing for FH be $\Delta \omega$ and the symbol time be $T$. For a rectangular chip pulse if we define the bandwidth of the DS signal as the location of the first spectral null, then $\kappa=T \Delta \omega$; this is the time-bandwidth product of the frequency hopping tone. As an example, for $\mathrm{FH}$ with a rectangular pulse shape if we assume $\Delta \omega=T^{-1}$ then $^{3} \kappa=1$. In the following plots we assume $\kappa-1$. In Fig. 2, we have plotted the capacities $C_{D}$ for the cases $L=100$ and $L=1000, C_{M}$, for $M=2,4,8,16$, and the bound $C_{O}$, versus the parameter $G$. The parameter $G$ behaves like an interference to signal ratio. For low

\footnotetext{
${ }^{3}$ With this minimum $\Delta \omega$ (for noncoherent orthogonality) the effect of splashes would be significant in the case of unsynchronized hopping. thus the results in the plots hold only for synchronized hopping. Similar plots can easily be obtained for unsynchronized hopping if the timebandwidth product $\kappa$ is made larger (e.g., $\kappa>3$ ) for a Gaussian pulse
} shape function. 


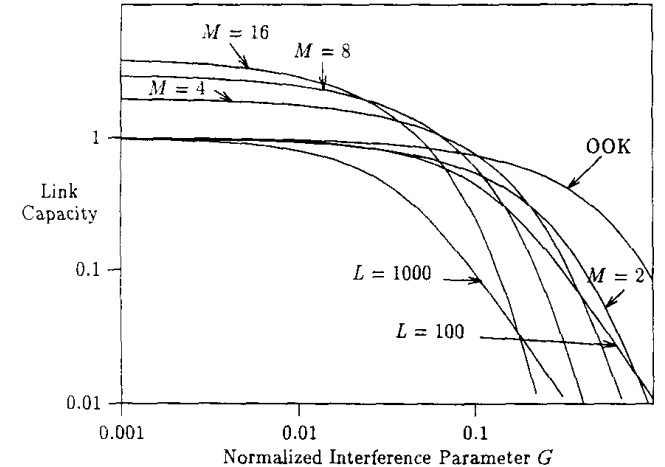

Fig. 2. Link capacity versus the normalized interference parameter $G$ for different signaling schemes.

values of $G, C_{O}$ and $C_{D}$ approach 1 and $C_{M}$ approaches $\log _{2} M$. However, as $G$ increases the performance of the $M$-ary schemes decreases drastically, and for $G \sim 1$ the OOK scheme is the best. For very large values of $G$ all capacities drop to zero; however, if we fix $L$ and let $G \rightarrow \infty$ then the DS/BPSK scheme is asymptotically better than all other schemes.

\section{DisCussion}

The link quality parameters, $\mu_{O}, \mu_{M}$, and $\mu_{D}$, determine the bandwidth (or processing gain) versus transmission range trade-off for the various schemes. These parameters incorporate the link distance $R$, the level of interference (in terms of $\lambda$ ), and the processing gain (in terms of $L$ and $Q$ ). The parameter $N$ in (24) is a dimensionless measure of the square of the link distance. For a fixed performance level $N$ is proportional to $\sqrt{L}$ in the case of DS /BPSK ( $L$ equals the number of data chips per data symbol), and for the frequency hopping schemes $N$ is proportional to $Q$, the number of orthogonal tones.

For a fixed level of interference and a large processing gain the capacity is not too small and the frequency hopping schemes perform better than the direct sequence scheme. For a fixed processing gain and large interference (large $N$, i.e., low link quality) the order is reversed. As an example, for DS/BPSK and $\mu_{D} \ll 1$ we obtain $C_{D} \sim$ $\mu_{D}^{2} /(\pi \ln 2)$, and for FH/BPSK and $\mu_{M} \ll 1$ we have $C_{2} \sim \mu_{M}^{4} /(128 \ln 2)$. Fixing $Q$ and letting $G \rightarrow \infty$, we obtain $C_{M} / C_{D} \rightarrow 0$.

In multihop packet radio networks, it is not fair to compare links in terms of the capacity if the link distances are not equal and a long link with low capacity may be better than a short link with high capacity. The overall traffic in the network, hence the required capacity, depends on the length of the transmission hops. As a general rule if the link distance is decreased by a given factor then the capacity requirement increases by the same factor, hence a good performance measure is the product of the link distance and link capacity. The parameter $G$ is proportional to the square of the distance, hence $\sqrt{G}$ is a measure of distance. We use the link performance mea-

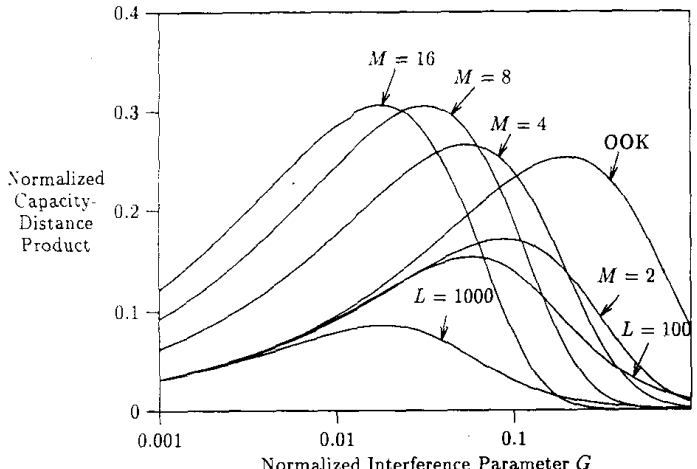

Fig. 3. Capacity-distance product versus the normalized interference parameter $G$ for different signaling schemes.

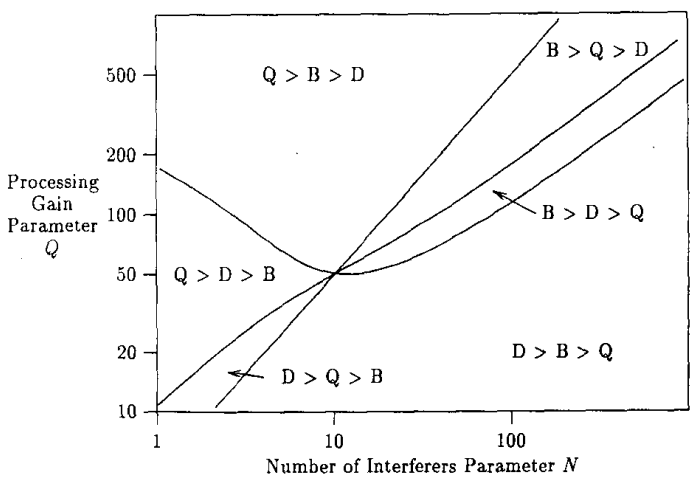

Fig. 4. Comparison of three signaling schemes: D: DS/BPSK, B FH/BFSK, Q: FH/4FSK (" $X>Y$ " indicates that, in the given region, $X$ performs better than $Y$ ).

sure $C \sqrt{G}$, where $C$ is the capacity of the link. In Fig. 3, we have plotted the product $C \sqrt{G}$, the capacity-distance product for the schemes considered in this paper.

To exhibit further the relative capacity of the previous schemes, we consider the schemes DS/BPSK, FH/BFSK, and FH/4FSK. The first two of these compare DS with $\mathrm{FH}$ whereas the last is an example of the effect of using a larger symbol alphabet. We may compare these by taking two at a time and finding the region in the $N-Q$ plane where one is better than the other. In Fig. 4, we have plotted 3 curves. Each curve compares two schemes and partitions the plane into two regions. The three curves partition the plane into six regions with each corresponding to a particular order of the capacity of the schemes. The three curves intersect at a point, a "triple point" where the three schemes have equal capacity.

From the previous statements, we may conclude that unless we are interested in "long" links with very small capacity, the frequency hopping schemes are better than the direct sequence scheme. There are two mechanisms that account for the degradation of link quality, the strength of the interferers, and the number of them. The direct sequence and frequency hopping schemes cope with these factors in different ways. For any processing gain, $L$, 
the capacity of a direct sequence link may be brought to zero, even if there is only one interferer, by increasing the signal strength of the interferer. The direct sequence scheme is mainly sensitive to the total interference power. In the case of frequency hopping, there is an upper bound to the degradation that may be caused by one interferer regardless of its power, and this upper bound decreases as the processing gain increases. The frequency hopping schemes are more sensitive to the number of interferers that have a significant power level. These observations are similar to those made in [14].

The capacity results presented were for the coding channel resulting from the hard decision detection scheme. Capacity results for the predetection channel (the waveform channel) were not given since they will depend on the exact form of the propagation amplitude loss function $a(r)$ for very small values of $r$. A propagation loss function that is more accurate for very small values of $r$ would be needed to find the capacity of the waveform channel; however, such a function would likely result in intractable expressions for the distribution of the interference.

\section{GeNERALIZATIONS}

In this paper, we have considered frequency hopping schemes with one symbol per hop. The analysis may be generalized to other spread spectrum schemes include the case of frequency hopping with more than one symbol per hop. Our restriction has been imposed by the requirement for spherical symmetry in the characteristic function $\Phi(\cdot)$ in proceeding beyond (12). To handle more general influence functions we retract to (9) and perform the integration with respect to $r$ first. In doing this, the integral with respect to $r$ is at first truncated to avoid convergence problems, and then the limit is taken. The result, assuming $\alpha=1$, is

$$
\psi(\boldsymbol{\omega})=-\lambda \frac{\pi^{2}}{2} \boldsymbol{E}\left(\left|\boldsymbol{\omega}^{T} \boldsymbol{X}\right|+j \frac{2}{\pi}\left(\boldsymbol{\omega}^{T} \boldsymbol{X}\right) \ln \left|\boldsymbol{\omega}^{T} \boldsymbol{X}\right|\right),
$$

where the expectation is over the influence function $\boldsymbol{X}$. This is the log-characteristic function for a class of multidimensional stable distributions of parameter $\alpha=1$ (see [12, pp. 19-20] for a more general form). For many cases of interest the probability density function of $\boldsymbol{X}$ is symmetric about the origin and the second of the previous terms vanishes. The log-characteristic function then simplifies to

$$
\psi(\boldsymbol{\omega})=-\lambda \frac{\pi^{2}}{2}\|\boldsymbol{\omega}\| \boldsymbol{E}\left(\left|\boldsymbol{\eta}^{T} \boldsymbol{X}\right|\right),
$$

where $\boldsymbol{\eta}$ is a vector on the unit sphere $(\|\boldsymbol{\eta}\|=1)$.

As an example, we consider the case of direct sequence with a very small processing gain and two symbol block (i.e., $n=2$ ). For simplification we assume chip synchronization. Thus, the influence function is $\boldsymbol{X}=\left(x_{1}, x_{2}\right)$, where $x_{i}=\sum_{k=1}^{L} c_{k, i}$, and the $c_{k, i}$ 's are binary i.i.d. random variables taking the values \pm 1 . The lack of spherical symmetry in the characteristic function leads to difficulty

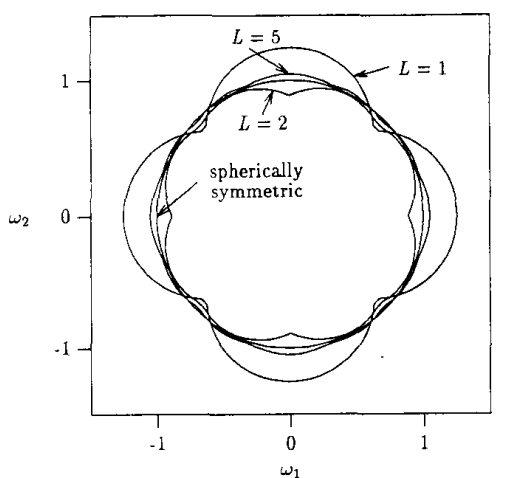

Fig. 5. Convergence of the characteristic function of a two-dimensional interference vector to a spherically symmetric function.

in obtaining the probability density. In some cases, we may simply approximate the probability density with one having spherical symmetry. To check the current example, in Fig. 5 we show plots of the normalized value $\sqrt{\pi / 2}\left(1 / \sigma_{x}\right) \boldsymbol{E}\left(\left|\boldsymbol{\eta}^{T} \boldsymbol{X}\right|\right)$, where $\sigma_{x}$ is the standard deviation of $x_{i}$, and compare it with the unit circle which corresponds to the case of $\boldsymbol{X}$ having a spherically symmetric density. As seen from the plots the curve converges quickly, as $L$ increases, to the unit circle. This assumption on chip synchronization is no burden since its removal will result in the probability density being a mixture of densities, and mixtures tend to preserve spherical symmetry.

In this paper, we assumed that the interferers are distributed in the plane according to a Poisson process with parameter $\lambda$. To obtain a finite interference we must also have $\gamma>2$. It is possible to generalize these assumptions slightly, with minimal effort, for centralized networks. If we assume that the receiver is at the origin and the interferers are Poisson distributed (with parameter $\lambda$ ) in a sector of angle $\theta_{s}$ then we may apply the preceding analysis with $\lambda$ reduced by the factor $\theta_{s} /(2 \pi)$. We may also consider the case of a Poisson process in the plane with nonuniform parameter $\lambda=\lambda(r)$. Let us assume that $\lambda(r)=\lambda_{0} r^{-\nu}$, where $0<\nu<2$. We may make a transformation on the plane $P$ to the plane $P^{\prime}$ as follows: In terms of polar coordinates, we let $r^{\prime}=r^{1-n u / 2}$ and $\theta^{\prime}=$ $\theta$. The Poisson process in the $P$ plane is mapped to a Poisson process in the $P^{\prime}$ plane with constant parameter $\lambda=2 \lambda_{0} /(2-\nu)$. If the propagation power loss exponent in $P$ is $\gamma$, then in $P^{\prime}$ the corresponding exponent is $\gamma^{\prime}=2 \gamma /(2-\nu)$. As an example, if we have $\nu=1$ then we can handle the case of free space $(\gamma=2)$ in $P$, since $\lambda^{\prime}=4>2$.

We draw the attention of the reader to the work in [14] were a similar approach to the one in this paper is used to find the performance of a link in the presence of a finite set of interferers whose positions are distributed according to a bell-shaped density function centered at the receiver. The form of the density of the terminals is specifically chosen so as to make the analysis tractable, and the assumption is made that the interference powers 
combine additively. We also refer the reader to [5], which has appeared after the initial submission of the current paper, where a FH/MFSK scheme is analyzed in the presence of a Poisson field of interferers. This paper assumes slow frequency hopping and considers the effect of thermal noise but makes more use of approximations.

\section{CONCLUSION}

We have considered a link in a radio network that is affected by a Poisson field of interfering transmitters. The Poisson model corresponds to the case of complete lack of knowledge of terminal positions. Under this model and a $1 / r^{4}$ power loss law we have shown that the probability law of the interference signal of a set of receiver correlator outputs is the multivariate Cauchy probability law. The probability of symbol error and the capacity versus the link distance was found for a set of signaling schemes utilizing spread spectrum. The method presented in this paper may be readily extended to obtain the performance of a link versus distance for other spread spectrum schemes.

\section{REFERENCES}

[1] R. Kahn, S. Gronemeyer, J. Burchfiel, and R. Kunzelman, "Advances in packet radio technology," Proc. IEEE, vol. 66, pp. 1468-1496, Nov. 1978

[2] V. H. MacDonald, "Advanced mobile phone service: The cellular concept," Bell Syst. Tech. J., vol. 58, no. 1, pp. 15-41, Jan. 1979.

[3] D. Verhulst, M. Mouly, and J. Szpirglas, "Slow frequency hopping multiple access for digital cellular radiotelephone," IEEE J. Select. Areas Commun., vol. 2, pp. 563-574, July 1984.

[4] J. E. Padgett, "Channel requirements for a cordless telephone spectrum allocation," IEEE J. Selec. Areas Commun., vol. 5, pp. 783-795, June 1987.

[5] J. W. Gluck and E. Geraniotis, "Throughput and packet error probability in cellular frequency-hopped spread-spectrum radio networks," IEEE J. Select. Areas Commun., vol. 7, pp. 148-160, Jan. 1989.

[6] M. K. Simon, J. K. Omura, R. A. Scholtz, and B. K. Levitt, Spread Spectrum Communications. Rockville, MD: Computer Science Press, 1984.

[7] E. A. Geraniotis and M. B. Pursley, "Error probabilities for slow-frequency-hopped spread-spectrum multiple-access communications over fading channels," IEEE Trans. Commun., vol. 30, pp. 996-1009, May 1982.

[8] E. S. Sousa, "Interference modeling in a direct sequence spread spectrum packet radio network," IEEE Trans. Commun., vol. 38, pp. $1475-1482$, Sept. 1990.

[9] - "On distributed spread spectrum packet radio networks," Ph.D. thesis, Dept. of Elect. Eng. Syst., Univ. of Southern California, Los Angeles, Dec. 1985.

[10] E. S. Sousa and J. A. Silvester, "Optimum transmission ranges in a direct sequence spread spectrum multi-hop packet radio network," IEEE J. Select. Areas Commun., vol. 8, pp. 762-771, June 1990.

[11] S. Chandrasekhar, "Stochastic problems in physics and astronomy," Rev. Modern Phys., vol. 15, no. 1, pp. 1-89, Jan. 1943.

[12] V. M. Zolotarev, One-Dimensional Stable Distributions, Translations of Mathematical Monographs. Providence, RI: American Mathematical Society, 1986, vol. 65.

[13] W. C. Jakes, Ed., Microwave Mobile Communications. New York: Wiley, 1974.

[14] S. A. Musa and W. Wasylkiwskyj, "Co-channel interference of spread spectrum systems in a multiple user environment," IEEE Trans. Commun., vol. COM-26, pp. 1405-1413, Oct. 1978. 